\title{
Caracterización del consumo de oxígeno en población especial de la Afeau
}

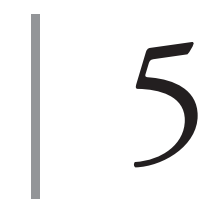

https://doi.org/10.21830/9789585241466.05

\author{
Angélica Maria Puentes Salazar \\ Oscar Mauricio Sarmiento Becerra ${ }^{2}$
}

\section{Resumen}

Objetivo: en este estudio se analizan los resultados de pruebas de consumo de oxígeno realizadas a personal de la Agrupación de Fuerzas Especiales Antiterroristas Urbanas (Afeau) de las Fuerzas Armadas de Colombia. Métodos y materiales: se trata de un estudio transversal que analiza los resultados de la prueba de ergoespirometría en tapiz rodante con un protocolo progresivo e incremental tipo Bruce modificado que se aplicó a dos grupos distintos de la Afeau en 2016 y 2018. Las pruebas fueron realizadas por personal del laboratorio del Centro de Investigación de la Cultura Física (Cicfi) de la Escuela Militar de Cadetes "General José María Córdova” (Esmic) en un Ergoespirómetro Metalyzer 3B-R2 y tapiz rodante marca HP Cosmos. Con la informat ción obtenida se realizó un análisis descriptivo y los datos se muestran como medianas y rangos intercuartílicos (RIC) dado el pequeño tamaño de la muestra. Resultados: el consumo mediano de oxígeno en el umbral y máximo de los participantes fue de 39 $(\mathrm{RIC}=37-45)$ y $45(\mathrm{RIC}=43-50) \mathrm{mL} / \mathrm{min} / \mathrm{kg}$, respectivamente. Por su parte, la frecuencia cardíaca mediana en el umbral fue de $155 \mathrm{Ipm}(\mathrm{RIC}=152-158)$, mientras que la mediana de la frecuencia máxima alcanzó los $174 \mathrm{Ipm}(\mathrm{RIC}=166-176)$. La velocidad máxima realizada en la prueba obtuvo una mediana de $18,5 \mathrm{~km} / \mathrm{h}(\mathrm{RIC}=18,5-$ 20,1). Conclusiones: los resultados mostraron que en general la población analizada de

1 Médico especialista en Medicina de la Actividad Física y del Deporte. Centro de Investigación de la Cultura Física (Cicfi), Escuela Militar de Cadetes "General José María Córdova”, Bogotá, D. C., Colombia. Contacto: angelica.puentes@esmic.com - http://orcid.org/0000-0002-2682-4185

2 Médico especialista en Medicina de la Actividad Física y del Deporte. Unidad de Ciencias Aplicadas al Deporte (UCAD) del Instituto distrital de recreación y deporte (IDRD). Contacto: omsarmiento@fucsalud.edu.co - http://orcid.org/0000-0002-9305-5822 
la Afeau tiene una adecuada condición cardiovascular y que supera los valores medios de la población general (ligeramente mejor en el grupo del 2018).

Palabras clave: ergoespirometria, consumo máximo de oxígeno, resistencia cardio vascular

\section{Introducción}

En este estudio se describe la resistencia cardiovascular como uno de los componentes de la aptitud física relacionados con la salud (1). Específicamente, se mide el consumo de oxígeno a través de la ergoespirometría en tapiz rodante en una población especial dentro de la Agrupación de Fuerzas Especiales Antiterroristas Urbanas (Afeau) de las Fuerzas Armadas Colombianas. La variable más importante en la realización de la prueba física es el consumo de oxígeno máximo, el cual se entiende como "la cantidad máxima de oxígeno que el organismo es capaz de absorber, transportar y consumir por unidad de tiempo" (2), y consiste en el producto del gasto cardíaco máximo (litros de sangre por minuto -1) y la diferencia de oxígeno arterio-venoso ( $\mathrm{mL}$ oxígeno por litros de sangre) (1).

Teniendo en cuenta que el consumo de oxígeno máximo se ha considerado un medio para evaluar las adaptaciones al entrenamiento de resistencia cardiovascular (3), caracterizar esta población permite tener una medición objetiva de su condición cardiovascular y hacer comparaciones con los resultados de consumo de oxígeno por ergoespirometrías de otras poblaciones militares. Entre los datos analizados en esta investigación se incluyó información sobre edad, talla y peso para tener un mejor conocimiento de la población. Asimismo, con base en los reportes de la ergoespirometría se evaluó consumo de oxígeno, frecuencia cardiaca y velocidad máxima en la prueba, datos que no solo ayudan a evaluar el grado de entrenamiento en relación con la condición cardiovascular, sino que además constituyen un insumo para plantear sugerencias al programa de entrenamiento.

De acuerdo con la literatura, el consumo de oxígeno máximo es un indicador de la capacidad funcional de un individuo y depende de diversos factores: la genética, que es el mayor condicionante, determina hasta el 70 
$\%$; la edad, el sexo, la masa y la composición corporal pueden afectar hasta el $50 \%$, mientras que el grado de entrenamiento o de acondicionamiento físico pueden mejorar los indicadores hasta en el $20 \%(2,3)$. Los valores esperados para hombres de entre 20 y 40 ańos son de 35 a $45 \mathrm{~mL} / \mathrm{kg} /$ min, y dependiendo del tipo de entrenamiento puede oscilar entre 40 y 80 $\mathrm{mL} / \mathrm{kg} / \mathrm{min}$ (2). También es importante resaltar que un componente del consumo de oxígeno es el gasto cardiaco, que se calcula como el producto de la frecuencia cardiaca y el volumen sistólico, los cuales aumentan con la actividad física. Por su parte, la respuesta de la frecuencia cardiaca depende de la edad y del entrenamiento: cuanto mayor es el entrenamiento, mayor es la respuesta (4), y es una de las variables que se miden durante toda la prueba de ergoespirometría.

\section{Metodología}

Es una descripción retrospectiva de los resultados de la prueba de ergoespirometría en tapiz rodante con un protocolo progresivo e incremental tipo Bruce modificado, que consta de tres etapas: 1) calentamiento (con velocidad constante de $4 \mathrm{~km} / \mathrm{h}$ y con inclinación de $1 \%$ durante dos minutos), 2) aplicación de los estadios (iniciando desde $7 \mathrm{~km} / \mathrm{h}$ con aumento de 1,6 km cada minuto, con inclinación constante de 1\%) y 4) recuperación (descenso progresivo de la velocidad hasta regresar a $4 \mathrm{~km} / \mathrm{h}$, manteniendo inclinación de 1\%). Estas pruebas fueron realizadas a dos grupos diferentes en dos periodos: el primero con cuatro integrantes, medidos en diciembre del 2016 y el segundo con cinco individuos en mayo de 2018. Dichas ergoespirometrías fueron tomadas en el laboratorio del Centro de Investigación de la Cultura Física (Cicfi) de la Escuela Militar de Cadetes "General José María Córdova" (Esmic) por el personal del grupo de investigación de la misma institución e interpretado por el médico del deporte del Cicfi. Las pruebas fueron realizadas en su totalidad por los integrantes de la Agrupación de Fuerzas Especiales Antiterroristas Urbanas (Afeau), pero no se incluyó una prueba del grupo de 2018 por ser parcial. Específicamente, 
se valoraron las siguientes variables: consumo de oxígeno en el umbral y el máximo; la frecuencia cardiaca al umbral y al máximo, y la velocidad máxima alcanzada en la prueba. El equipo utilizado fue el Ergoespirómetro Metalyzer 3B-R2 y tapiz rodante marca HP Cosmos. Con la información obtenida se realizó un análisis descriptivo en el programa estadístico Stata 13. Los datos se muestran como medianas y rangos intercuartílicos (RIC) dado el pequeño tamaño de muestra.

\section{Resultados}

Se incluyeron nueve miembros de la Afeau durante los periodos 2016 y 2018, a quienes se les midió consumo de oxígeno con ergoespirometría en tapiz rodante. En el año 2016 se incluyó el 44.44\% de la población y en 2018 el $55.56 \%$

La mediana de edad de los participantes fue de 34 años $(\mathrm{RIC}=31-34)$, la mediana de la talla y el peso fue 1,69 metros $(\mathrm{RIC}=1,66-1,72)$ y 69 kilogramos $(\mathrm{RIC}=66-72)$, respectivamente. En la tabla 1 se resumen las características básicas de la población de estudio dividida según el año (2016 y 2018).

Tabla 1. Características de la población

\begin{tabular}{cccccc}
\hline Año & Variable & Mediana & RIC* & Mínimo & Máximo \\
\hline \multirow{2}{2}{016} & Talla (m) & 1,68 & $1,63-1,73$ & 1,6 & 1,76 \\
& Peso (Kg) & 67,5 & $62,0-70,5$ & 58 & 72 \\
& Edad (años) & 34 & $31,5-36,5$ & 29 & 39 \\
$\mathbf{2 0 1 8}$ & Talla (m) & 1,69 & $1,66-1,72$ & 1,65 & 1,76 \\
& Peso (Kg) & 69 & $67,0-74,0$ & 61 & 75 \\
& Edad (años) & 33 & $31,0-34,0$ & 29 & 36 \\
\multirow{2}{*}{ Total } & Talla (m) & 1,69 & $1,66-1,72$ & 1,6 & 1,76 \\
& Peso (Kg) & 69 & $66,0-72,0$ & 58 & 75 \\
& Edad (años) & 34 & $31,0-34,0$ & 29 & 39 \\
\hline
\end{tabular}

* RIC: rango intercuartílico.

Fuente: Original de los autores. 
Los individuos medidos durante el año 2018 presentaron una menor mediana de edad (figura 1), pero a su vez una mayor mediana de talla (figura 2) y peso (figura 3).

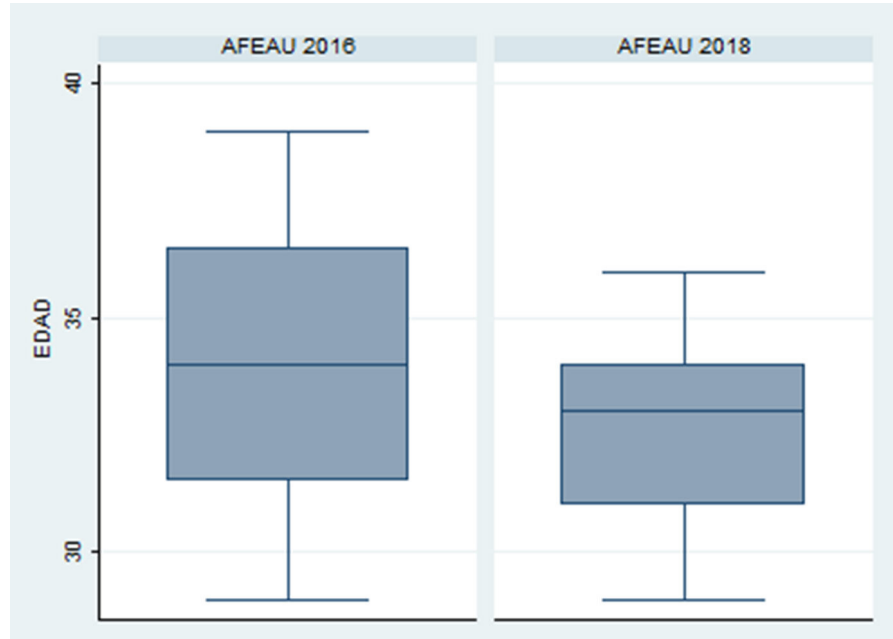

Figura 1. Distribución de la edad (años) según el año de ingreso al estudio. Fuente: Original de los autores.

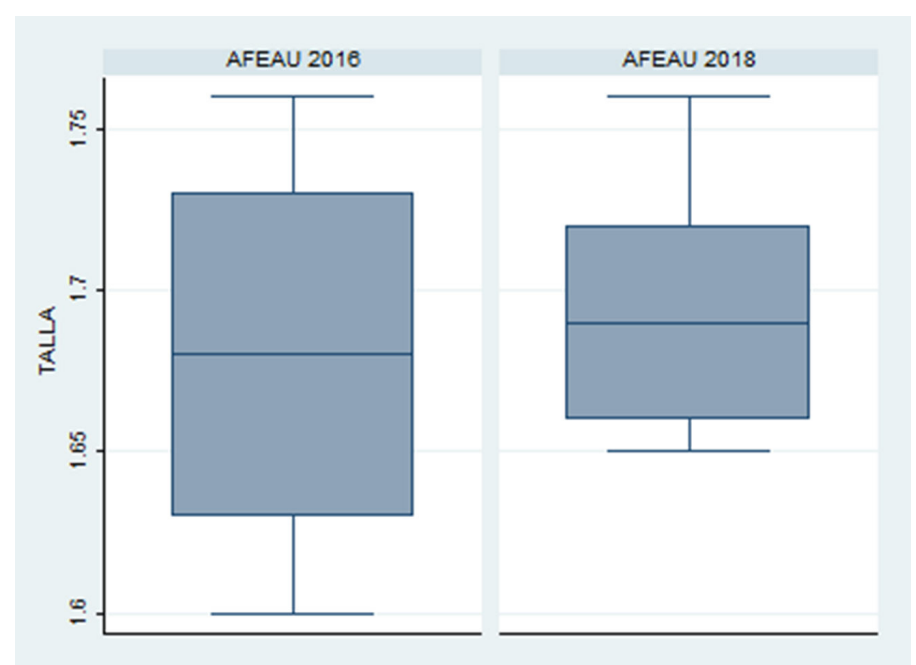

Figura 2. Distribución de la talla $(\mathrm{m})$ según el año de ingreso al estudio. Fuente: Original de los autores. 


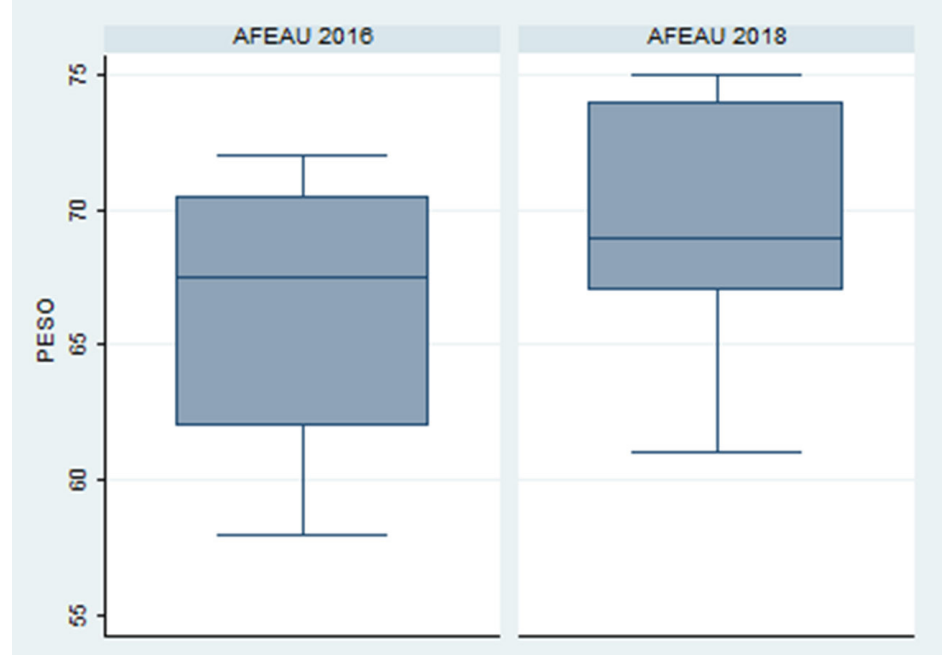

Figura 3. Distribución del peso $(\mathrm{kg})$ según el año de ingreso al estudio. Fuente: Original de los autores.

En la tabla 2 se presentan las mediciones de la condición respiratoria del consumo de oxígeno por ergoespirometría. Aquí se puede observar en el total de la población que el consumo mediano de oxígeno en el umbral y máximo de los participantes fue de $39(\mathrm{RIC}=37-45)$ y $45(\mathrm{RIC}=43-50)$ $\mathrm{mL} / \mathrm{min} / \mathrm{kg}$, respectivamente. Por su parte, la frecuencia cardíaca mediana en el umbral fue de $155 \mathrm{Ipm}$ (RIC = 152-158), mientras que la mediana de la frecuencia máxima alcanzó los 174 Ipm (RIC = 166-176). La velocidad máxima realizada en la prueba obtuvo una mediana de $18,5 \mathrm{~km} / \mathrm{h}(\mathrm{RIC}=$ 18,5-20,1).

El grupo de participantes del año 2018 obtuvo mayores valores medianos del consumo de oxígeno en el umbral (figura 4) y máximo (figura 5), comparado con los participantes del 2016. 
Tabla 2. Características clínicas

\begin{tabular}{|c|c|c|c|c|c|}
\hline Año & Variable & Mediana & RIC* & Mínimo & Máximo \\
\hline \multirow[t]{5}{*}{2016} & $\mathrm{VO}_{2}$ umbral $(\mathrm{mL} / \mathrm{min} / \mathrm{kg})$ & 38 & $36,5-40,5$ & 36 & 42 \\
\hline & $\mathbf{V O}_{2}$ máximo $(\mathrm{mL} / \mathbf{m i n} / \mathbf{k g})$ & 44,5 & $43,0-48,0$ & 42 & 51 \\
\hline & FC umbral (Ipm) & 158,5 & $152,0-169,0$ & 152 & 173 \\
\hline & FC máxima (Ipm) & 176,5 & $166,5-179,0$ & 159 & 179 \\
\hline & Velocidad máxima $(\mathrm{km} / \mathrm{h})$ & 18,5 & $18,5-19,3$ & 18,5 & 20,1 \\
\hline \multirow[t]{5}{*}{2018} & $\mathrm{VO}_{2}$ umbral $(\mathrm{mL} / \mathrm{min} / \mathrm{kg})$ & 45 & $37,0-46,0$ & 36 & 46 \\
\hline & $\mathrm{VO}_{2}$ máximo $(\mathrm{mL} / \mathrm{min} / \mathrm{kg})$ & 48 & $43,0-50,0$ & 41 & 53 \\
\hline & FC umbral (Ipm) & 155 & $152-158$ & 152 & 158 \\
\hline & FC máxima (Ipm) & 168 & $166,0-175,0$ & 159 & 176 \\
\hline & Velocidad máxima $(\mathrm{km} / \mathrm{h})$ & 20,1 & $18,5-20,1$ & 16,9 & 20,1 \\
\hline \multirow[t]{5}{*}{ Total } & $\mathrm{VO}_{2}$ umbral $(\mathrm{mL} / \mathrm{min} / \mathrm{kg})$ & 39 & $37,0-45,0$ & 36 & 46 \\
\hline & $\mathrm{VO}_{2}$ máximo $(\mathrm{mL} / \mathrm{min} / \mathrm{kg})$ & 45 & $43,0-50,0$ & 41 & 53 \\
\hline & FC umbral (Ipm) & 155 & $152,0-158,0$ & 152 & 173 \\
\hline & FC máxima (Ipm) & 174 & $166,0-176,0$ & 159 & 179 \\
\hline & Velocidad máxima $(\mathrm{km} / \mathrm{h})$ & 18,5 & $18,5-20,1$ & 16,9 & 20,1 \\
\hline
\end{tabular}

* RIC: rango intercuartílico.

Fuente: Original de los autores.

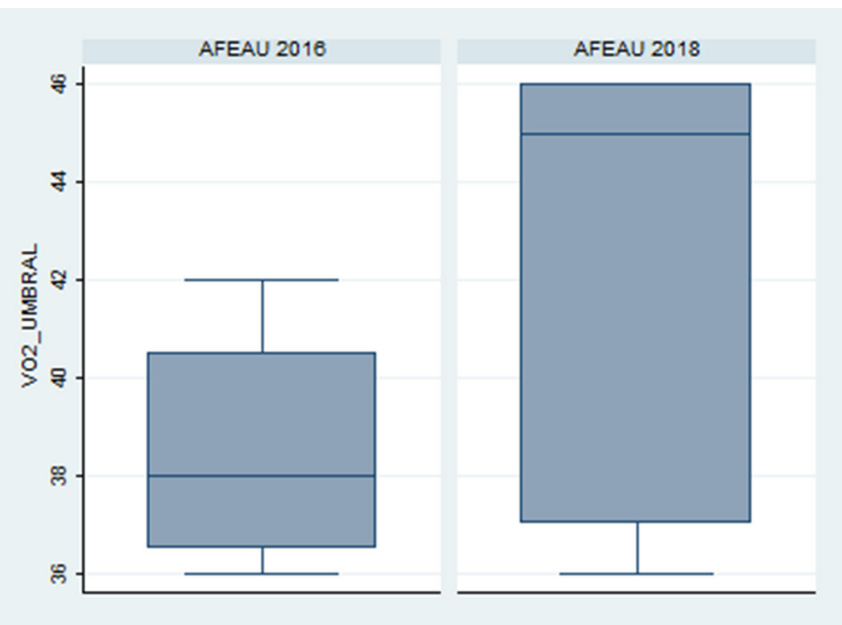

Figura 4. Distribución del consumo de oxígeno en el umbral $(\mathrm{mL} / \mathrm{min} / \mathrm{kg})$ según el año de ingreso al estudio.

Fuente: Original de los autores. 


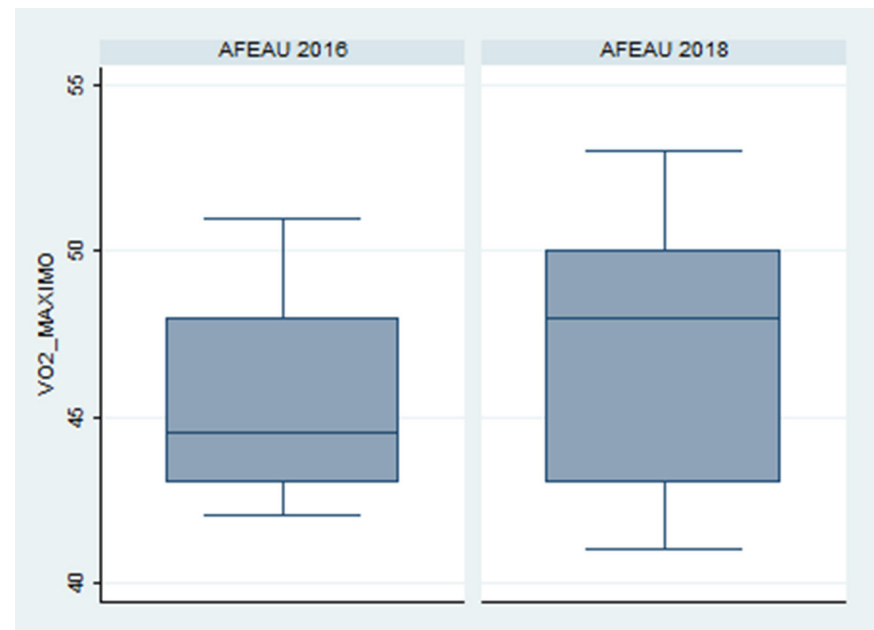

Figura 5. Distribución del consumo de oxígeno máximo $(\mathrm{mL} / \mathrm{min} / \mathrm{kg})$ según el año de ingreso al estudio.

Fuente: Original de los autores.

Por su parte, el grupo de participantes del año 2016 obtuvo mayores valores medianos de la frecuencia cardíaca en el umbral (figura 6) y máxima (figura 7).

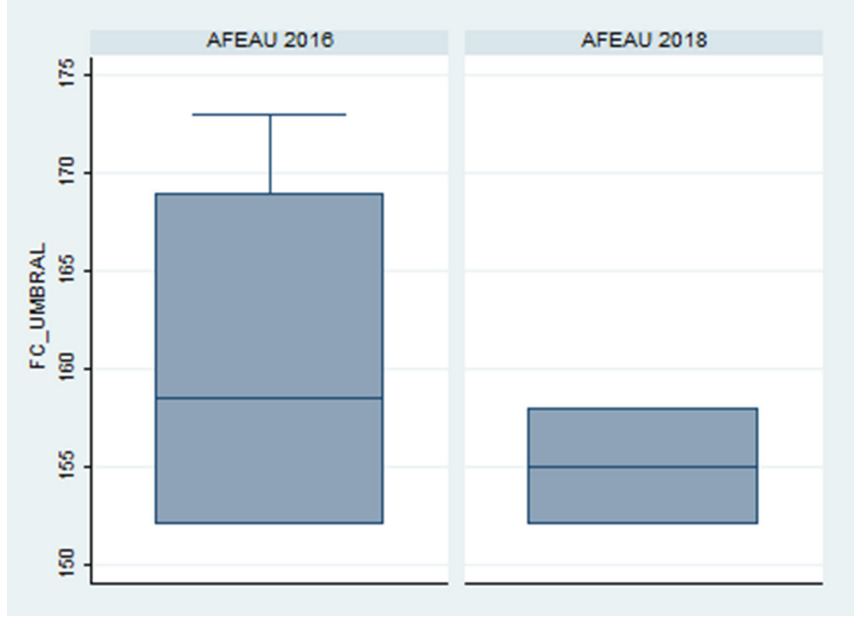

Figura 6. Distribución de la frecuencia cardíaca en el umbral (Ipm) según el año de ingreso al estudio.

Fuente: Original de los autores. 


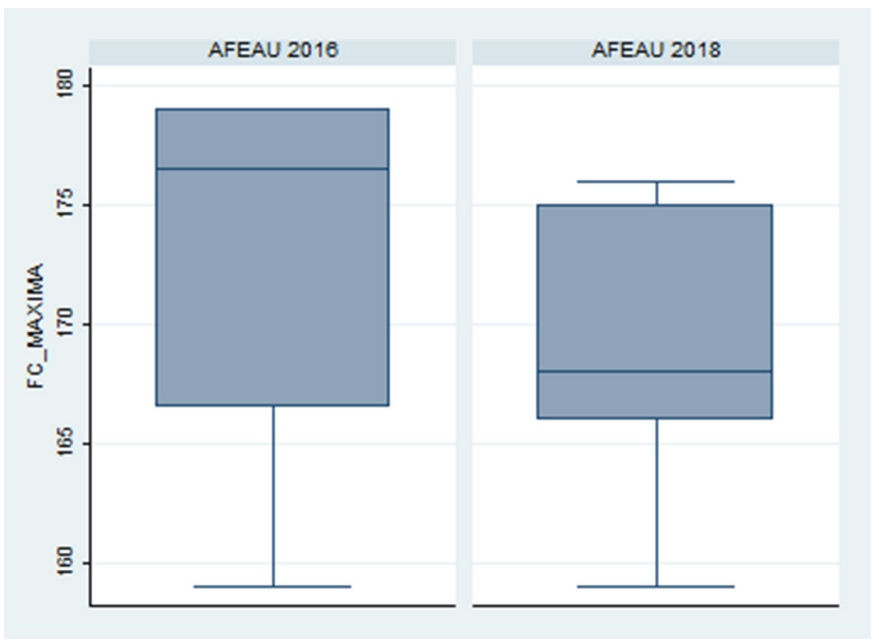

Figura 7. Distribución de la frecuencia cardíaca máxima (Ipm) según el año de ingreso al estudio.

Fuente: Original de los autores.

Otro parámetro que se evaluó con los datos obtenidos del consumo de oxígeno y de la frecuencia cardiaca es el segundo umbral ventilatorio representado por el porcentaje de los valores maximales en los cuales se cumplen los criterios fisiológicos que nos indican un cambio en el predominio de metabolismo glucolítico a oxidativo, esto con el fin de dar recomendaciones de trabajo en el entrenamiento para mejorar la resistencia cardiovascular. Respecto al consumo de oxígeno se observó que los valores de los porcentajes al umbral fueron todos superiores al $81 \%$, mientras que los porcentajes de la frecuencia cardiaca máxima — que es la más utilizada para hacer modificaciones en el entrenamiento por su facilidad en la monitorización - en que se logró el umbral fueron superiores al $86 \%$, con 6 de las 9 mediciones por encima del $90 \%$.

\section{Discusión}

Las dos agrupaciones evaluadas tienden a ser similares en edad, talla y peso, con resultados también similares de consumo de oxígeno máximo por 
rangos intercuartílicos de 43-48 mL / kg / min en el grupo Afeau 2016 y de 43-50 mL / kg / min en el grupo Afeau 2018. Los valores se encuentran ligeramente por encima de los rangos esperados $(35-45 \mathrm{~mL} / \mathrm{kg} / \mathrm{min})$ en hombres sanos de 20 a 40 ańos (2), pero con diferencias en las medianas de $44,5 \mathrm{~mL} / \mathrm{kg} / \mathrm{min}$ para el grupo Afeau 2016 y de $48 \mathrm{~mL} / \mathrm{kg} / \mathrm{min}$ para el Afeau 2018, lo cual muestra una mejor condición física cardiovascular en el grupo de 2018. Con respecto al consumo de oxígeno al umbral, que es el punto de cambio metabólico entre lo estable y lo inestable (5), hay diferencia tanto en las medianas como en los rangos intercuartílicos con mediana de $38 \mathrm{~mL} / \mathrm{kg} / \mathrm{min}$ con RIC de 36,5-40,5 mL / kg/ min para el grupo de Afeua del 2016 y mediana de $45 \mathrm{~mL} / \mathrm{kg} / \mathrm{min}$ con RIC de $37-46 \mathrm{~mL} / \mathrm{kg}$ /min para el grupo de Afeua del 2018, lo cual evidencia que el grupo del 2018 tiene mejor condición cardiovascular. Esta cualidad es de suma importancia, pues en esta población el desempeño efectivo de sus tareas incluye diferentes destrezas y capacidades mentales y físicas. Tal hallazgo coincide con la revisión de literatura, en el sentido de que una adecuada aptitud física cardiovascular, al igual que programas de fuerza (6-8), les permiten a los individuos poner a prueba su entrenamiento, tanto en las tareas comunes, como en competencias militares internacionales, así como lograr sus tareas y objetivos (7-9).

Asimismo, se debe tener en cuenta que en este estudio solamente se hizo una medición del consumo de oxígeno máximo y al umbral de esta población cuando ya había cumplido su entrenamiento, es decir, no se cuenta con mediciones iniciales previas a su proceso de formación física. Esta circunstancia limita la posibilidad de evaluar el impacto de la preparación física recibida, como se reporta en estudios con población que presta el servicio militar obligatorio. En dichas investigaciones, las mediciones pre y post entrenamiento reportan que el acondicionamiento físico puede considerarse bajo si se quiere mejorar la resistencia en el personal militar, pues se encontró que el $\mathrm{VO}_{2 \text { max. }}$ aumentó $5 \%$ solo en los sujetos con el $\mathrm{VO}_{2 \max .}$ inicial bajo, sin cambios en los sujetos con $\mathrm{VO}_{2 \text { max. }}$ medios, mientras que en los sujetos con el nivel inicial más alto tendieron a disminuir (10). A diferencia del ante- 
rior, otro estudio con 72 reclutas sí reportó mejoría significativa del $\mathrm{VO}_{2 \max }$. con un entrenamiento básico de ocho semanas combinado con ejercicios de resistencia o entrenamiento de fuerza (11).

Finalmente, cabe señalar que así como Canadá, Holanda, Reino Unido y Estados Unidos han caracterizado su población militar en relación con el entrenamiento y han señalado la importancia de una adecuada condición física (7), esta investigación sobre la condición cardiovascular con medición del consumo de oxígeno máximo por medio de ergoespirometría de una población especial de la Afeau hace aportes que contribuyen al conocimiento y comprensión de la población militar colombiana.

\section{Conclusiones}

Los resultados mostraron que en general la población analizada de la Afeau tiene una adecuada condición cardiovascular y que supera los valores medios de la población general (ligeramente mejor en el grupo del 2018). No obstante, se desconoce el impacto del entrenamiento que recibe porque no se cuenta con una línea de base. En este sentido, es necesario conocer el tipo y las cargas de entrenamiento, comparar los resultados con otros parámetros como composición corporal y mediciones del consumo de oxígeno antes del inicio del entrenamiento y con seguimiento en el tiempo, lo cual sería de gran importancia en caracterizaciones futuras.

\section{Referencias}

1. Riebe D, Ehrman JK, Liguori G., Magal M. ACSM's guidelines for exercise testing and prescription from american college of sports medicine. Editorial Philadelphia: Wolters Kluwer; 2018.

2. López Chicharro J, Fernández Vaquero A. Fisiología del ejercicio. 3. ${ }^{a}$ ed. Bogotá, D. C.: Editorial Médica Panamericana; 2006. 1005 p.

3. Kenney WL, Wilmore JH, Costill DL. Fisiología del deporte y el ejercicio. 5. a ed. Bogotá, D. C.: Editorial Médica Panamericana; 2014. 575 p.

4. Narvani AA, Thomas P, Lynn B. Key topics in sports medicine. Reino Unido: Routledge Taylor \& Francis, 2006. 
5. Serrato Roa M. Medicina del deporte. 1. ${ }^{a}$ ed. Bogotá, D. C.: Universidad del Rosario. 2008.

6. Harman EA, Gutekunst DJ, Frykman PN, Nindl BC, Alemany JA, Mello RP, Sharp MA. Effects of two different eight-week training programs on military physical performance. J Strength Con Res. 2008 marzo; 22 (2): 524-534. DOI: http://dx.doi. org/10.1519/JSC.0b013e31816347b6

7. Task Group 019. Optimizing operational physical fitness. North Atlantic Treaty Organisation. 2009 enero. Reporte No: RTO-TR-HFM-080. Disponible en: http:// citeseerx.ist.psu.edu/viewdoc/download?doi=10.1.1.214.8364\&rep=rep1\&type=pdf

8. Vickers RR, Amanda Jr, Barnard C. Effects of physical training in military populations: a meta-analytic summary. San Diego, (CA): Naval Health Research Center; 2010 octubre 10. Reporte No: NHRC-11-17. Disponible en: https://pdfs.semanticscholar. org/62c8/3a11c39b9401acb8a066885e467831479f0a.pdf

9. Ejército Nacional de Colombia. Mejor equipo fuerzas comando 2018, categoría imagen institucional. Revista Ejército, 2018; 199. Disponible en: https://dicoe.mil.co/revista_ ejercito/revista/Revista_199/mejor-equipo-fuerzas-comando-2018.html

10. Dyrstad SM, Soltvedt R, Hallén J. Physical fitness and physical training during Norwegian military service. Mil. Med. 2006 agosto; 171 (8): 736-741. DOI: http:// dx.doi.org/10.7205/milmed.171.8.736

11. Santtila M, Häkkinen K, Karavirta L, Kyröläinen H. Changes in Cardiovascular Performance during an 8-week military basic training period combined with added endurance or strength training. Mil. Med. 2008 diciembre; 173 (12): 1173-1179. DOI: http://dx.doi.org/10.7205/milmed.173.12.1173 